\title{
Flambagem local e global de vigas de aço formadas a frio com seção ponto-simétrica $Z$ sob flexão oblíqua
}

\author{
Janderson Leitão Sena ${ }^{1}$ e Eduardo de Miranda Batista ${ }^{1 *}$ \\ ${ }^{1}$ Programa de Engenharia Civil, COPPE, Universidade Federal do Rio de Janeiro, \\ Rio de Janeiro, RJ, Brasil. CP 68506, 21945-972. \\ dersonlsena@coc.ufrj.br; batista@coc.ufrj.br
}

\section{Local and global buckling of Z-section cold-formed steel beams under oblique flexural bending}

\begin{abstract}
Resumo
O presente trabalho apresenta formulações para a flambagem global e local em seções abertas de paredes finas, do tipo Z, que encontram larga utilização como perfis de aço formados a frio. Para o modo local, foram obtidos os coeficientes de flambagem local de seções $Z$ sob flexão oblíqua, sendo essa condição não considerada na norma brasileira ABNT NBR 14762:2010. Para o modo global, constatou-se que a equação de momento crítico sugerida na referida norma não atende as equações diferenciais da Teoria da Estabilidade Elástica apresentadas por TIMOSHENKO e GERE (1961). Nesse contexto, os autores apresentam solução adequada, que atende a solução teórica acima citada. Ao final, é apresentada uma tabela comparativa dos diferentes modos de obtenção do momento crítico global, considerando a equação da norma, a equação desenvolvida pelos autores e as análises numéricas segundo o MFF e da GBT.
\end{abstract}

Palavras-chave: Perfis de aço formados a frio; seção Z; flexão oblíqua; flambagem global; flambagem local.

\begin{abstract}
The present work presents formulations for global and local buckling of $Z$ thin-walled sections, with wide application as cold-formed steel members. For the local mode, the buckling local coefficients of $Z$ sections under oblique flexion were obtained, considering this condition is not considered in the Brazilian code ABNT NBR 14762:2010. For the global mode, it was found that the critical bending moment equation suggested in the Brazilian code does not meet the differential equations of Elastic Stability Theory presented by TIMOSHENKO and GERE (1961). In this context, the authors present an adequate solution, which meets the theoretical solution mentioned above. Finally, the different ways of obtaining the global critical bending moment are compared, considering the equation of the Brazilian code, the equation developed by the authors and the numerical analysis results according to FSM and the GBT.
\end{abstract}

Keywords: Steel cold-formed member; Z section; oblique flexural bending; global buckling; local buckling.

\footnotetext{
* Autor correspondente
} 


\section{Introdução}

A utilização de perfis de aço formados a frio (PFF), também conhecidos como perfis de chapa dobrada, tem larga utilização na construção civil. Entre as vantagens que seu uso proporciona destacam-se a alta relação resistência/peso das estruturas, sistemas estruturais leves, menor tempo de fabricação, transporte e montagem, melhor aproveitamento de espaço no canteiro de obra, além da facilidade na obtenção de uma grande variedade de seções abertas, garantindo assim uma maior liberdade arquitetônica em projeto.

As seções abertas de paredes finas são especialmente sensíveis aos fenômenos de flambagem, com destaque para efeitos de torção, flambagem local e distorcional. A flambagem afeta e contribui diretamente para a redução do esforço resistente de PFF, podendo ocorrer segundo os modos local, distorcional e global. Logo, identificar os possíveis modos de flambagem e obter os seus respectivos esforços críticos é um aspecto fundamental para o dimensionamento de estruturas constituídas por perfis de aço formados a frio.

O uso de PFF permite a obtenção de diferentes geometrias, sendo muito utilizadas em projeto as seções do tipo monossimétrica, duplamente simétrica e simétrica em relação a um ponto, esta última também conhecida por ponto-simétrica. $\mathrm{O}$ uso destes perfis abrange uma vasta gama de possibilidades, em especial na composição de coberturas metálicas, verificando-se a aplicação de seções ponto-simétricas do tipo Z, em particular, como elementos de terças, conforme representado na Figura 1.

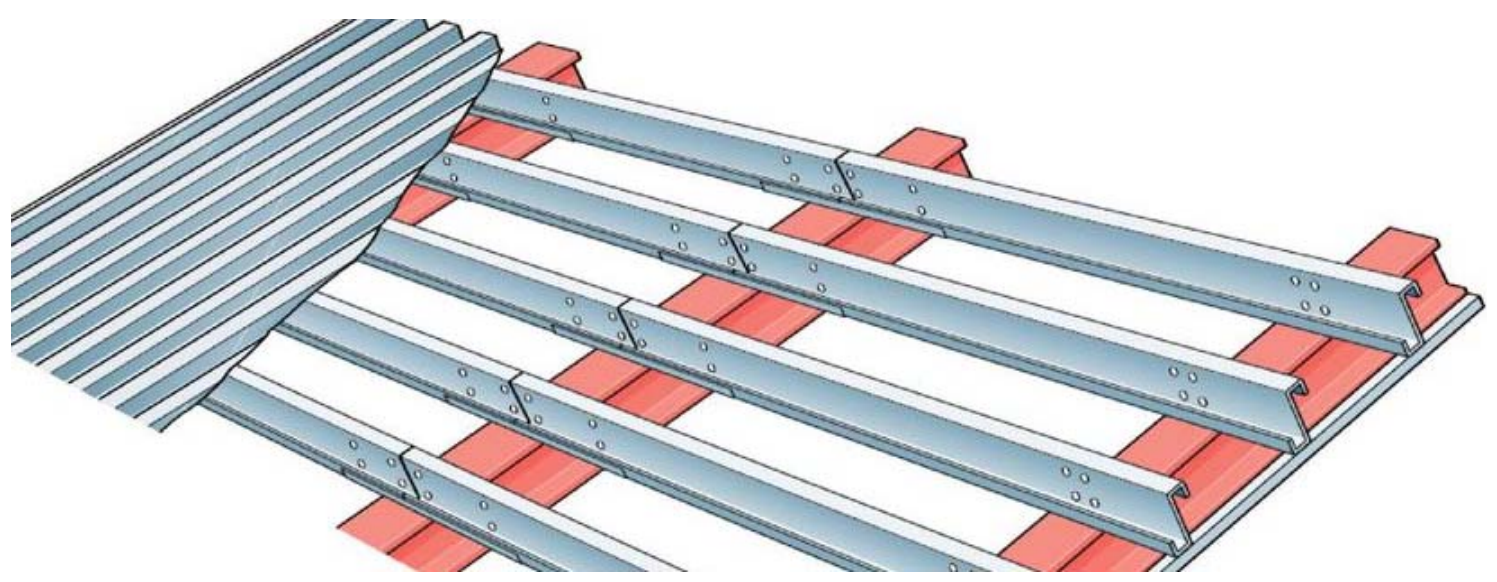

Figura 1 - Terças de aço constituídas por PFF de seção ponto-simétrica Z enrijecida (PEREIRA, 2016) 
As terças de cobertura são componentes que trabalham essencialmente à flexão, sendo o dimensionamento para a referida solicitação prescrito nas normas técnicas, as quais tratam de garantir a segurança estrutural. Entre os métodos de dimensionamento mais usuais estão o Método da Seção Efetiva (MSE) e o Método da Resistência Direta (MRD), ambos presentes na ABNT NBR 14762:2010. O primeiro foi proposto por BATISTA (2010) e incorporado à norma brasileira em sua última versão. O segundo foi originalmente desenvolvido por SCHAFER (2006), e, além de compor a ABNT NBR 14762:2010, encontra-se também na norma americana AISI S100-16 (2016).

Conforme a necessidade em projeto, a opção por seções ponto-simétricas Z em detrimento das seções monossimétricas $U$ sob flexão justifica-se por sua geometria favorecer o armazenamento, transporte e principalmente o transpasse nas ligações, como pôde ser observado na Figura 1. Além disso, os perfis de seção Z apresentam característica favorável quando carregados no plano da alma, visto que neste elemento estão localizados, por questões geométricas, o centróide e o centro de torção da seção transversal. Logo, para forças externas alinhadas com o centróide (e centro de torção), a barra não está sujeita a momento torsor.

\section{Objetivos}

A presente investigação trata, em particular, dos modos de flambagem local e global, em seções ponto-simétricas $Z$ simples ou enrijecida, sob flexão simples em torno do eixo perpendicular à alma. O principal objetivo é verificar os procedimentos para dimensionamento estrutural constantes na ABNT NBR 14762:2010, visando a segurança e economia em projeto estrutural, levando-se em conta a condição conflitante de tratar a flexão como oblíqua ou restringida. A primeira condição decorre da flexão livre da barra como uma viga sem elementos de contenção lateral no vão, enquanto a segunda se refere à condição usual de terças restringidas pela fixação às telhas, somada à contenção lateral proporcionada por linhas de corrente.

Para o modo local serão apresentados os coeficientes de flambagem local $k$ para seções completas sob flexão oblíqua. Já para o modo global a contribuição da pesquisa fica por conta da elaboração de uma equação para o cálculo do momento crítico em seções duplamente simétricas e ponto-simétricas, baseada na Teoria da Estabilidade Elástica 
apresentada por TIMOSHENKO e GERE (1961). A Figura 2 ilustra os referidos modos de flambagem em uma seção $Z$ enrijecida sob flexão.
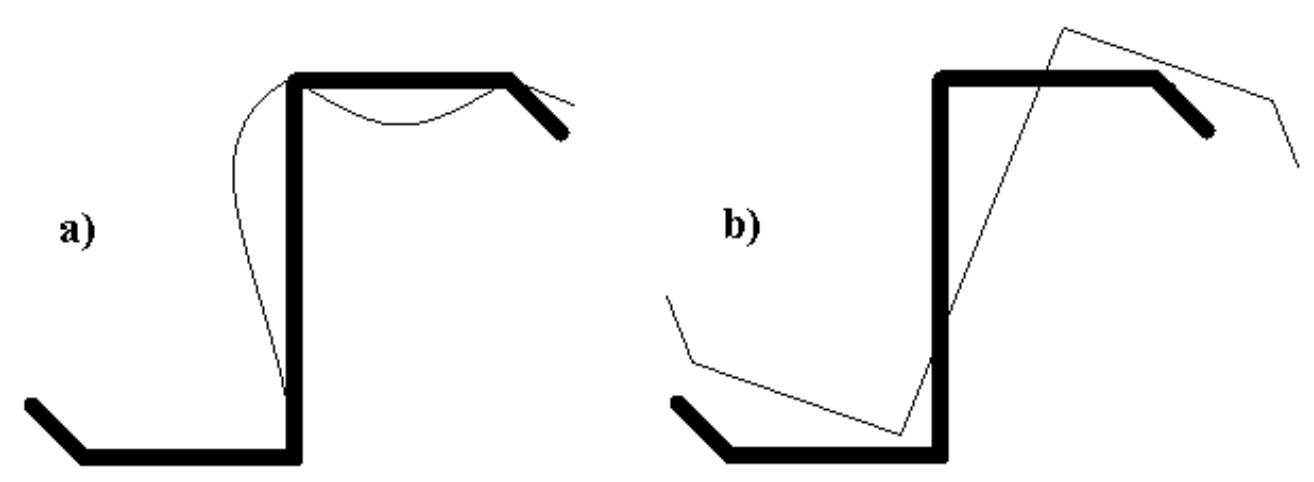

Figura 2 - Modos de flambagem em seções ponto-simétricas do tipo $Z$ enrijecido na flexão: (a) modo local (b) modo global de flambagem lateral com torção

Apesar da ABNT NBR 14762:2010 apresentar tabelas e expressões que permitem obter o coeficiente de flambagem local kpara seções completas na flexão, assim como dispõe de equações para o cálculo do momento crítico global, a elaboração do presente trabalho tem por objetivo complementar propor o aprimoramento das prescrições da norma brasileira, visto que as proposições aqui tratadas são distintas e, julgamos, mais adequadas do que aquelas inseridas na versão atual da norma brasileira, para o caso de seções do tipo $Z$.

\section{$3 \quad$ Flexão restringida e Flexão livre}

Em seções do tipo U, o eixo de flexão, normal à alma, define a simetria da seção, sendo igualmente o eixo principal de inércia máximo. Para o caso de seções $Z$, o eixo normal à alma não será principal de inércia, seja a seção simples ou enrijecida. Logo, de acordo com as condições em que se encontra o perfil de seção $Z$ na estrutura, a flexão em torno deste eixo pode ser tratada de dois modos diferentes: como flexão restringida (lateralmente) ou flexão livre (obliqua), sendo a primeira condição mais usual em projeto, em especial quando se trata de terças de coberturas com águas inclinadas.

Conforme referido anteriormente, a flexão restringida se desenvolve em torno de um eixo que não é principal de inércia da seção. Em virtude do deslocamento da seção estar impedido na direção do eixo de flexão, devido à condições proporcionadas por vínculos externos, ocorre o que pode ser definido como um caso de flexão reta "forçada". Logo, 
o referido eixo assume o papel de eixo principal da seção, fazendo com que a distribuição de tensões normais na seção se manifeste para a situação na qual a linha neutra coincide com o referido eixo, similar a um eixo principal.

A Figura 3 ilustra a distribuição de tensões normais em seções ponto-simétricas Z segundo a flexão restringida (reta) e flexão livre (oblíqua), quando solicitadas por carregamento externo alinhado com o plano da alma.

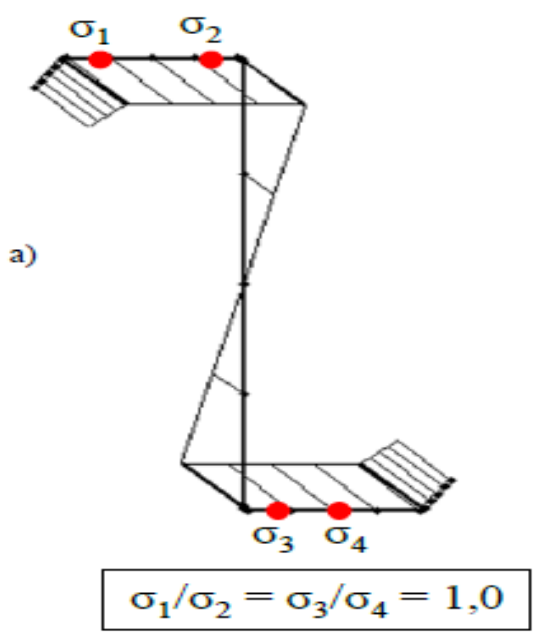

b)

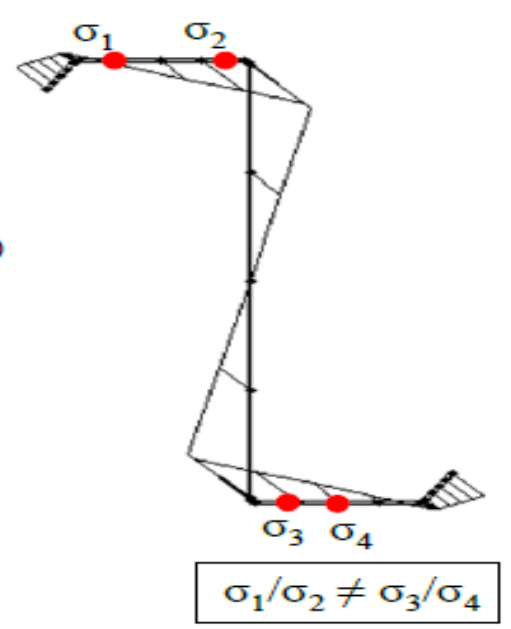

Figura 3 - Distribuição de tensões em seção Z: (a) flexão restringida (b) flexão livre (FÁVERO, 2013)

O estudo da flexão livre em seções ponto-simétricas $Z$ sob flexão em torno do eixo perpendicular à alma da seção foi recentemente tratado por FÁVERO (2013). Em seu trabalho sobre ligações em terças, o autor apresentou resultados teóricos e experimentais que comprovaram a distribuição de tensões em seções $Z$ mais próximas da flexão oblíqua se comparada à flexão reta "forçada". Além disso, esse autor constatou que o dimensionamento baseado na flexão livre pode ser considerado mais adequado quando comparado com a condição de flexão restringida. As observações apresentadas pelo autor motivaram a elaboração da presente pesquisa, adotando-se a flexão livre em seções ponto-simétricas $Z$.

\section{$4 \quad$ Modo de flambagem local}

A equação para o cálculo da tensão crítica de flambagem local $\sigma_{c r}$ presente na ABNT NBR 14762:2010, considerando seções completas, é equivalente à equação utilizada para o cálculo da tensão crítica local em placas isoladas, definida segundo a Teoria da 
Estabilidade Elástica apresentada por TIMOSHENKO e GERE (1961), sendo expressa pela Equação [1]:

$$
\sigma_{c r}=k \ell \cdot\left[\frac{\pi^{2} \cdot E}{12 \cdot\left(1-v^{2}\right)}\right] \cdot\left(\frac{t}{b_{w}}\right)^{2}
$$

Entre os termos que compõem a Equação [1], diferem do caso da análise de placas isoladas o valor de $b_{w}$ e do coeficiente de flambagem local $k_{\text {. }}$ Para placas isoladas os valores adotados são da largura $b$ do elemento e o respectivo coeficiente de flambagem, de acordo com as condições de contorno e tipo de carregamento na placa. Já para o caso de seções completas (caso dos PFF, em particular), os valores inseridos na equação são do elemento de maior largura da seção, no caso a alma $b_{w}$, e o coeficiente de flambagem local $k$, considerando a seção completa.

O cálculo da tensão crítica local considerando propriedades da seção completa, em substituição ao Método da Largura Efetiva (MLE), foi proposto por BATISTA (2010), originando o Método da Seção Efetiva (MSE) constante da ABNT NBR 14762:2010. O referido método permite um dimensionamento muito mais expedito e preciso, se comparado ao tradicional MLE, lembra BATISTA (2010), já que o método não trata elementos isolados, mas sim seções completas e a consequente interação entre as paredes da seção. Apesar de sua praticidade para o cálculo manual, para que se obtenha o coeficiente de flambagem local $k$ k de seções completas é necessário o uso de programas computacionais especializados em análise da Estabilidade Elástica. Devido a esta necessidade, a norma brasileira apresenta os valores do coeficiente $k$, na forma de tabelas e equações para as seções mais usuais, dentre elas as seções ponto-simétricas Z aqui investigadas.

As Tabelas 12 e 13 da ABNT NBR 14762:2010, que disponibilizam o cálculo e a consulta, respectivamente, dos coeficientes de flambagem local k/para seções completas na flexão simples, considerando seções ponto-simétricas Z carregadas no plano da alma, satisfazem apenas a condição de flexão restringida, enquanto a presente investigação aborda a flexão livre. A constatação baseou-se nas verificações efetuadas com o auxílio do programa computacional CUFSM v.3.12, representando o Método das Faixas Finitas 
(MFF) e desenvolvido por SCHAFER e ÁDÁNY (2006). De acordo com os valores obtidos numericamente, foram consideráveis as diferenças encontradas comparando-se com os valores apresentados na norma brasileira de PFF, tratando a flexão como livre. Entretanto, quando verificados para a condição de flexão restringida, os valores coincidiram com aqueles constantes na ABNT NBR 14762:2010.

Conclui-se que a inclusão das seções ponto-simétricas $Z$ nas Tabelas 12 e 13 da norma é limitada ao caso de flexão restringida, não havendo informação que esclareça essa condição: os coeficientes de flambagem local $k$, satisfazem apenas a condição de flexão restringida. Além disso, o eixo perpendicular à alma da seção, no caso de seções pontosimétricas Z, não se trata do eixo de maior inércia, sendo a referência das tabelas válidas apenas para o caso das seções monossimétricas $U$.

Logo, em complemento à ABNT NBR 14762:2010, foram analisadas numericamente com o auxílio do programa CUFSM v.3.12, seções ponto-simétricas Z, simples e enrijecidas, sob a condição de flexão livre em torno do eixo perpendicular à alma, e, com a contribuição da Equação [1], foram obtidos os valores dos coeficientes de flambagem local $k$,na flexão oblíqua. Para os perfis enrijecidos foram investigadas seções limitadas à relação (enrijecedor/alma) $D / b_{w}=0,3$, seguindo, assim, limitação apresentada na ABNT NBR 14762:2010. Foram adotadas seções enrijecidas a $90^{\circ}$, porém sendo desprezível a diferença para o caso de enrijecedores de borda a $45^{\circ}$. A Tabela 1 apresenta os valores dos coeficientes de flambagem local $k$, obtidos.

Após a obtenção dos coeficientes de flambagem, foi elaborada a superfície de tendência referente aos valores de $k$, em função das relações geométricas $b_{f} / b_{w}$ e $D / b_{w}$. A superfície obtida é ilustrada na Figura 4.

Observando a Figura 4, nota-se uma superfície pouco irregular enquanto existe a presença de enrijecedores de borda $\left(D / b_{w}>0\right)$, porém uma variação abrupta ocorre quando se aproxima da geometria da seção $Z$ simples $\left(D / b_{w}=0\right)$. Logo, foi necessário separar as expressões para cada caso, mantendo-se assim uma boa precisão para os valores da análise numérica. Para os perfis simples foi utilizada função de uma variável apenas $\left(\mathrm{X}=b_{f} / b_{w}\right)$, representada pela Equação [2]. Já para os perfis $Z$ enrijecidos foi necessária uma função de duas variáveis $\left(\mathrm{X}=b_{f} / b_{w}\right.$ e $\left.\mathrm{Y}=D / b_{w}\right)$, representada pela Equação [3]. 
Tabela 1 - Valores dos coeficientes de flambagem local $k$, para seção $Z$, simples e enrijecida, sob flexão oblíqua, para momento fletor aplicado no plano da alma

\begin{tabular}{|c|c|c|c|c|c|c|c|}
\hline \multirow{2}{*}{$\mathbf{b}_{\mathbf{f}} / \mathbf{b}_{\mathbf{w}}$} & \multicolumn{7}{|c|}{$\mathbf{D} / \mathbf{b}_{\mathbf{w}}$} \\
\cline { 2 - 8 } & $\mathbf{0 , 0 0}$ & $\mathbf{0 , 0 5}$ & $\mathbf{0 , 1 0}$ & $\mathbf{0 , 1 5}$ & $\mathbf{0 , 2 0}$ & $\mathbf{0 , 2 5}$ & $\mathbf{0 , 3 0}$ \\
\hline $\mathbf{0 , 2}$ & 29,21 & 31,97 & 32,56 & 32,75 & 32,84 & 32,89 & 32,92 \\
\hline $\mathbf{0 , 3}$ & 29,91 & 31,20 & 31,42 & 31,50 & 31,54 & 31,55 & 30,19 \\
\hline $\mathbf{0 , 4}$ & 29,61 & 30,26 & 30,32 & 30,31 & 30,28 & 30,22 & 26,39 \\
\hline $\mathbf{0 , 5}$ & 22,20 & 28,97 & 28,87 & 28,73 & 28,58 & 28,38 & 23,82 \\
\hline $\mathbf{0 , 6}$ & 15,37 & 27,12 & 26,68 & 26,24 & 25,77 & 25,24 & 21,98 \\
\hline $\mathbf{0 , 7}$ & 11,28 & 24,25 & 23,43 & 22,65 & 21,90 & 21,14 & 20,34 \\
\hline $\mathbf{0 , 8}$ & 8,64 & 20,78 & 19,78 & 18,89 & 18,08 & 17,30 & 16,56 \\
\hline $\mathbf{0 , 9}$ & 6,83 & 17,49 & 16,53 & 15,69 & 14,95 & 14,26 & 13,62 \\
\hline $\mathbf{1 , 0}$ & 5,55 & 14,72 & 13,89 & 13,16 & 12,52 & 11,93 & 11,38 \\
\hline$A$
\end{tabular}

A relação $\mathrm{D} / \mathrm{b}_{\mathrm{w}}$ igual a zero representa os perfis ponto-simétricos $\mathrm{Z}$ simples (não enrijecidos) Para valores intermediários é sugerido interpolar linearmente

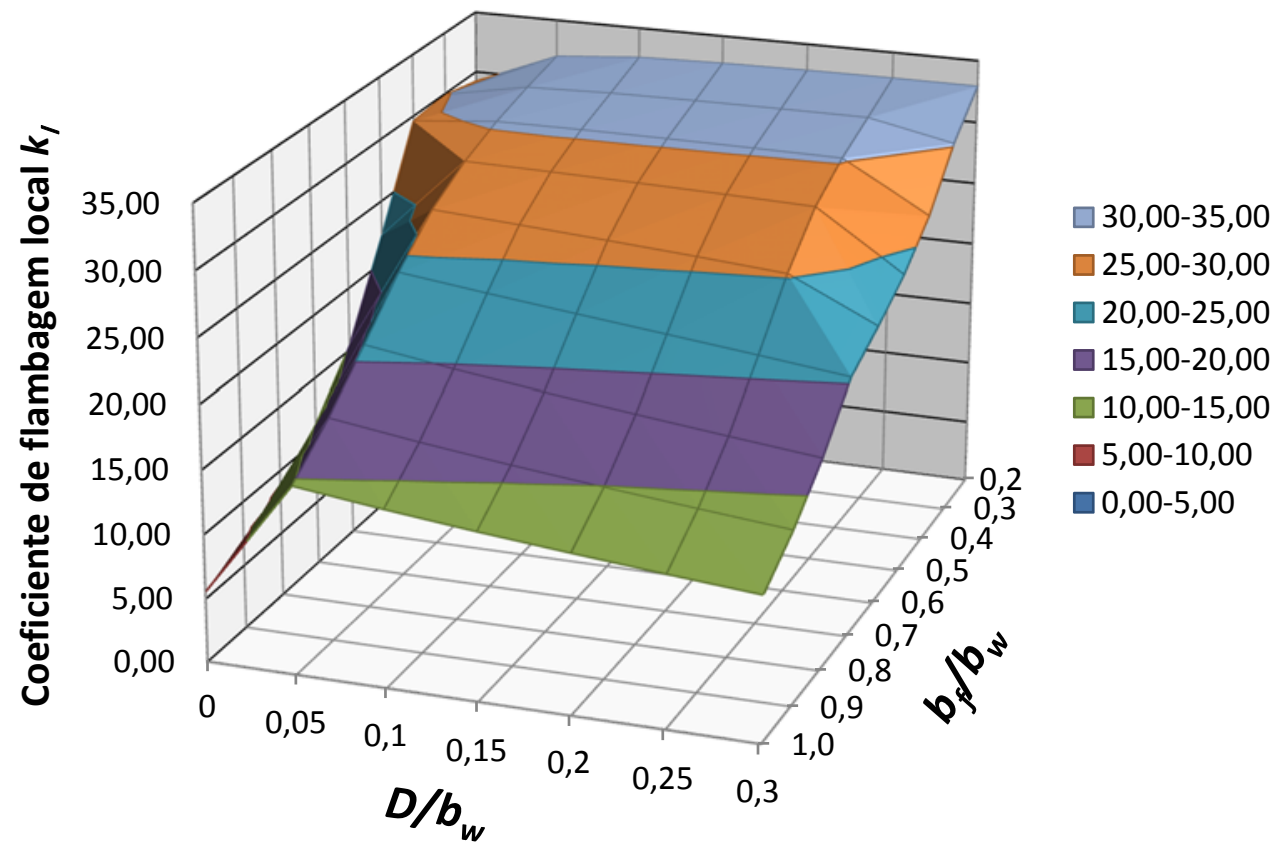

Figura 4 - Superfície formada pelos valores dos coeficientes de flambagem local $k$, para seção $Z$, simples e enrijecida, sob flexão oblíqua, em função das relações geométricas entre as larguras da alma, mesas e enrijecedor $\left(b_{w}, b_{f}\right.$ e $\left.D\right)$

$$
\begin{aligned}
& k_{l}=7574,5 X^{6}-28007 X^{5}+40919 X^{4}-29783 X^{3}+11165 X^{2}-2033,5 X+170,5 \\
& k_{l}=25,84+29,59 Y+126,45 Y^{2}-495,04 Y^{3}+39,16 X- \\
&-159,13 X Y+140,33 X Y^{2}-72,79 X^{2}+75,65 X^{2} Y+24,33 X^{3}
\end{aligned}
$$


É importante ressaltar que os valores dos coeficientes de flambagem local $k$ / obtidos no presente estudo, apresentados na Tabela 1 e disponibilizados também segundo as Equações [2] e [3], são referentes à flexão livre (oblíqua). Portanto, as demais propriedades da seção devem, obrigatoriamente, ser consideradas sob a mesma condição para um possível dimensionamento em estado limite último.

A Equação [1] para o cálculo da tensão crítica local permanece válida para a flexão livre ou restringida, sendo apenas necessário o cuidado de adotar o valor apropriado do coeficiente k/em cada caso. Contudo, para o cálculo do momento crítico local $M$ ideve ser aplicada a Equação [4], sendo necessário adotar o módulo de flexão elástica $W_{c}$ (referente ao bordo comprimido da seção; em posição, portanto, sujeita ao efeito da flambagem local). Observar que, neste caso, $W_{c}$ será distinto para a flexão livre ou restringida, não devendo, de forma alguma, serem confundidos.

$$
M_{\ell}=k_{\ell} \cdot\left[\frac{\pi^{2} \cdot E}{12 \cdot\left(1-v^{2}\right)}\right] \cdot\left(\frac{t}{b_{w}}\right)^{2} \cdot W_{c}
$$

\section{$5 \quad$ Modo de flambagem global}

Para o caso particular da flexão simples em seções ponto-simétricas Z bi-apoiadas, tomando-se um trecho compreendido entre seções contidas lateralmente e analisadas globalmente para carregamento transversal agindo no plano da alma, a ABNT NBR 14762:2010, assim como a norma americana AISI S100-16, define o cálculo do momento crítico global $M_{e}$, também conhecido por momento fletor de flambagem lateral com torção, segundo a Equação [5]:

$$
M_{e}=0,5 \cdot C_{b} \cdot r_{0}\left(N_{e y} \cdot N_{e z}\right)^{0,5}
$$

Sendo $N_{e y}$ e $N_{e z}$ as forças axiais de flambagem global elástica por flexão em torno do eixo principal y e por torção pura em torno do eixo longitudinal $z$, respectivamente. Para estas considerações, o eixo principal $x$ refere-se ao eixo de simetria em seções monossimétricas. A constante $r_{0}$ consiste no raio de giração polar da seção bruta em relação ao centro de torção, enquanto $C_{b}$ é o fator de modificação para momento fletor não uniforme, sendo este adotado ao longo de todo o estudo igual a 1,0, e, portanto, 
suprimido nas formulações que serão apresentadas. Os termos e propriedades geométricas citados podem ser conferidos diretamente na ABNT NBR 14762:2010, já que as nomenclaturas adotadas na presente pesquisa seguem o padrão da norma brasileira. Na Figura 5 estão identificados os elementos da seção tipo Z: $b_{w}$ alma, $b_{f}$ flanges ou mesas e $D$ enrijecedor. A espessura das paredes é referida por $t$.

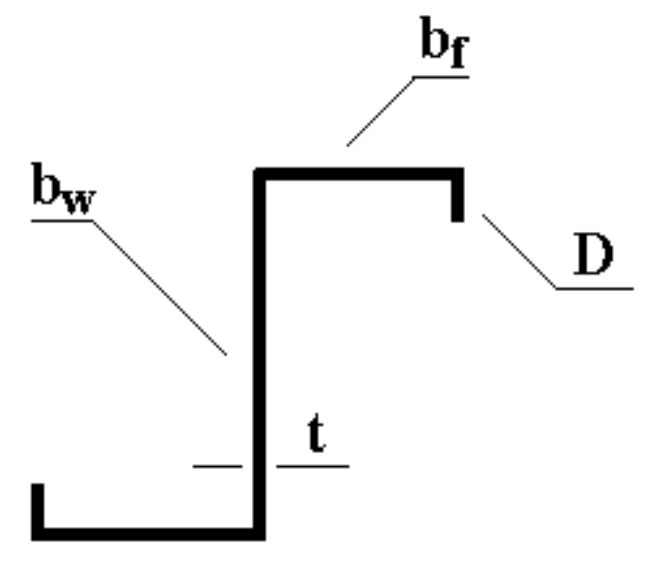

Figura 5 - Seção Z: identificação dos elementos de placa como as mesas, alma e enrijecedor e representação da espessura das paredes

Analisando-se previamente algumas seções ponto-simétricas $Z$ na condição de flexão oblíqua em torno do eixo perpendicular à alma, mais uma vez com o auxílio do programa CUFSM v.3.12, foram constatadas diferenças exorbitantes nos valores encontrados para $M_{e}$ em relação aos da equação da norma brasileira, representada pela Equação [5] na presente pesquisa. Logo, procurou-se compreender o motivo para resultados tão discrepantes partindo das formulações da Teoria da Estabilidade Elástica apresentadas por TIMOSHENKO e GERE (1961), considerando o caso mais básico da flexão (flexão pura, isto é, barra submetida a momento fletor constante).

Considere uma viga bi-apoiada, de comprimento $L$, sob momento fletor constante agindo em cada um dos eixos principais de inércia de sua seção, representados por $M_{1}$ e $M_{2}$, em referência aos eixos principais 1 e 2, respectivamente eixos máximo e mínimo, como ilustrado na Figura 6. Ressalta-se que nos apoios o deslocamento no plano da seção (eixos $x-y$, respectivamente 1-2) é impedido (contida lateralmente nas extremidades) e o empenamento é livre. As formulações apresentadas são válidas para carregamento externo alinhado com o centro de torção, similar à norma brasileira. 


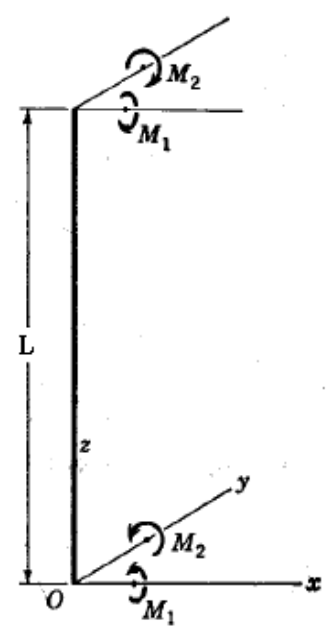

Figura 6 - Viga bi-apoiada solicitada por momentos fletores constantes ao longo da barra, de flexão segundo os planos principais de inércia, $M_{1}$ e $M_{2}$ (TIMOSHENKO e GERE, 1961)

O sistema de equações diferenciais para o cálculo do momento fletor de flambagem lateral com torção $M_{e}$, segundo a Teoria da Estabilidade Elástica apresentada por TIMOSHENKO e GERE (1961), é descrito pelas Equações [6], [7] e [8]. Os termos $u$ e $v$ representam respectivamente as translações nas direções principais $x$ e $y$ da seção, enquanto o termo $\phi$ refere-se ao ângulo de torção da seção em torno do eixo longitudinal z. Para efeito das formulações que serão apresentadas, os eixos $x$ e $y$ representam, respectivamente, os eixos principais de inércia máximo e mínimo da seção, eixos 1 e 2 .

$$
\begin{gathered}
E I_{y} \cdot \frac{d^{4} u}{d z^{4}}-M_{1} \cdot \frac{d^{2} \emptyset}{d z^{2}}=0 \\
E I_{x} \cdot \frac{d^{4} v}{d z^{4}}-M_{2} \cdot \frac{d^{2} \emptyset}{d z^{2}}=0 \\
E C_{\omega} \cdot \frac{d^{4} \emptyset}{d z^{4}}-\left(G J-M_{1} \cdot \beta_{1}-M_{2} \cdot \beta_{2}\right) \cdot \frac{d^{2} \emptyset}{d z^{2}}-M_{1} \cdot \frac{d^{2} u}{d z^{2}}+M_{2} \cdot \frac{d^{2} v}{d z^{2}}=0
\end{gathered}
$$

Sendo $I_{x}$ e $I_{y}$ os momentos principais de inércia máximo e mínimo da seção, respectivamente. Como propriedades da seção transversal têm-se a constante de empenamento $C_{\omega}$ e a constante de torção de Saint Venant $J$. Para o material têm-se os módulos de Elasticidade longitudinal $E$ e transversal $G$. Todos os termos aqui descritos são conhecidos e simples de serem obtidos, podendo ser conferidos também em bibliografia especializada. 
Complementando os termos apresentados, existem, ainda, os parâmetros geométricos da seção transversal $\beta_{1}$ e $\beta_{2}$, empregados exclusivamente no cálculo da flambagem lateral com torção. 0 primeiro está relacionado ao eixo $x$ (máximo, 1) e o segundo ao eixo y (mínimo, 2). O cálculo desses parâmetros, segundo TIMOSHENKO e GERE (1961), é definido pelas Equações [9] e [10], onde $x_{0}$ e yo representam a distância entre o centróide e o centro de torção da seção na direção de seus eixos principais de inércia.

$$
\begin{aligned}
& \beta_{1}=\frac{1}{I_{x}}\left(\int_{A}^{A} y^{3} d A+\int_{A}^{A} x^{2} y d A\right)-2 y_{0} \\
& \beta_{2}=\frac{1}{I_{y}}\left(\int_{A}^{A} x^{3} d A+\int_{A}^{A} y^{2} x d A\right)-2 x_{0}
\end{aligned}
$$

Estes parâmetros estão inteiramente associados à posição do centro de torção em relação aos eixos principais de inércia da seção. O valor do parâmetro $\beta$ se aproxima de zero à medida que o centro de torção está mais próximo do eixo de referência, ou seja, $\beta_{1}$ será igual a zero caso o centro de torção esteja localizado exatamente sobre o eixo 1 , assim como $\beta_{2}$ será nulo quando o centro de torção estiver localizado sobre o eixo 2 . Esta situação particular, inclusive, ocorre para as seções duplamente simétricas e as seções ponto-simétricas aqui investigadas, devido à posição do centro de torção coincidir com a posição do centróide. Logo, ambos os parâmetros, $\beta_{1}$ e $\beta_{2}$, assim como os demais termos que os acompanham na Equação [8], desaparecem do sistema de equações apresentado, o que vem a simplificar os cálculos posteriores.

Considerando, então, o caso particular das seções ponto-simétricas, suponha-se uma seção Z enrijecida solicitada por momento fletor $M$ aplicado em um eixo centroidal qualquer da seção, normal ou não à alma, defasado do ângulo $\theta$ para com o eixo principal de inércia máxima (eixo 1), como apresentado na Figura 7. Nessa figura é apresentado o caso mais usual de momento fletor aplicado no plano da alma, que inclusive se trata do caso de flexão investigado no presente estudo. 


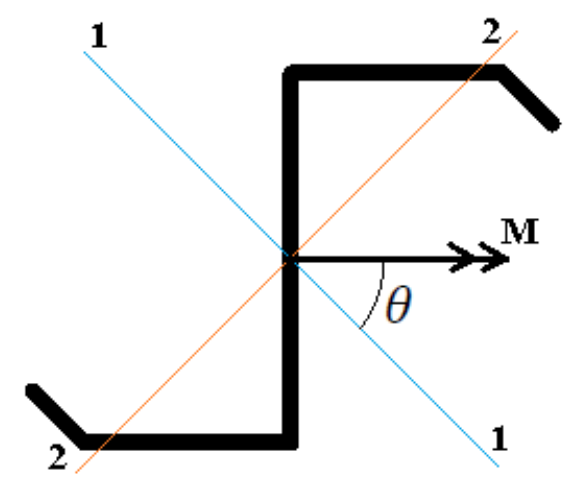

Figura 7 - Momento fletor $M$ agindo em um eixo centroidal qualquer, normal ou não à alma da seção ponto-simétrica $Z$

O momento fletor, por ser uma grandeza vetorial, pode ser decomposto nas direções dos eixos principais 1 e 2, os quais são perpendiculares entre si. Logo, o valor do momento crítico global $M_{e}$ pode ser definido segundo as expressões abaixo:

$$
M_{1}=M_{e} \cdot \cos \theta \quad M_{2}=M_{e} \cdot \operatorname{sen} \theta
$$

Portanto, novamente as equações diferenciais que governam a flambagem lateral com torção são introduzidas, fazendo-se agora a substituição das incógnitas $M_{1}$ e $M_{2}$ por uma única incógnita, o momento crítico global $M_{e}$, juntamente do ângulo de defasagem $\theta$ para com o eixo 1, dando origem às Equações [11], [12] e [13]:

$$
\begin{gathered}
E I_{y} \cdot \frac{d^{4} u}{d z^{4}}-M_{e} \cdot \cos \theta \cdot \frac{d^{2} \emptyset}{d z^{2}}=0 \\
E I_{x} \cdot \frac{d^{4} v}{d z^{4}}-M_{e} \cdot \operatorname{sen} \theta \cdot \frac{d^{2} \emptyset}{d z^{2}}=0 \\
E C_{\omega} \cdot \frac{d^{4} \emptyset}{d z^{4}}-G J \cdot \frac{d^{2} \emptyset}{d z^{2}}-M_{e} \cdot \cos \theta \cdot \frac{d^{2} u}{d z^{2}}+M_{e} \cdot \operatorname{sen} \theta \cdot \frac{d^{2} v}{d z^{2}}=0
\end{gathered}
$$

Os resultados da flambagem lateral com torção dependem, ainda, das condições de extremidade (vínculos) da barra. Para a condição estabelecida de apoios simples e empenamento livre, TIMOSHENKO e GERE (1961) definem como solução para as equações diferenciais a adoção de $u, v$ e $\phi$ segundo as expressões seguintes:

$$
u=A_{1} \cdot \operatorname{sen} \frac{\pi z}{L} \quad v=A_{2} \cdot \operatorname{sen} \frac{\pi z}{L} \quad \varnothing=A_{3} \cdot \operatorname{sen} \frac{\pi z}{L}
$$


Substituindo-se as expressões anteriores nas equações diferenciais do sistema, definidas pelas Equações [11], [12] e [13], e reorganizando-se os termos, obtêm-se as Equações [14], [15] e [16]:

$$
\begin{gathered}
\frac{\pi^{2} \cdot E I_{y}}{L^{2}} \cdot A_{1}+M_{e} \cdot \cos \theta \cdot A_{3}=0 \\
\frac{\pi^{2} \cdot E I_{x}}{L^{2}} \cdot A_{2}-M_{e} \cdot \operatorname{sen} \theta \cdot A_{3}=0 \\
M_{e} \cdot \cos \theta \cdot A_{1}-M_{e} \cdot \operatorname{sen} \theta \cdot A_{2}+\left(\frac{\pi^{2} \cdot E C_{\omega}}{L^{2}}+G J\right) \cdot A_{3}=0
\end{gathered}
$$

Uma solução possível para as equações do sistema seria considerar $A_{1}=A_{2}=A_{3}=0$, correspondente à configuração de equilíbrio indeformada ou inicial. Logo, para a configuração de equilíbrio deformada associada à flambagem lateral com torção, é preciso obter a solução que corresponde ao determinante nulo do sistema formado pelas Equações [14], [15] e [16]. Antes disso, os termos que compõem as equações do sistema podem ser simplificados, o que vem a facilitar as manipulações algébricas posteriores. Para isso são adotadas notações idênticas às da ABNT NBR 14762:2010, com o intuito de reduzir o número de termos das equações e facilitar o entendimento por parte do leitor:

$$
N_{e x}=\frac{\pi^{2} \cdot E I_{x}}{\left(K_{x} \cdot L_{x}\right)^{2}} \quad N_{e y}=\frac{\pi^{2} \cdot E I_{y}}{\left(K_{y} \cdot L_{y}\right)^{2}} \quad N_{e z}=\frac{1}{r_{0}^{2}} \cdot\left[\frac{\pi^{2} \cdot E C_{\omega}}{\left(K_{z} \cdot L_{z}\right)^{2}}+G J\right]
$$

Os termos $N_{e x}$ e $N_{e y}$ representam a força axial de flambagem global elástica por flexão em torno dos eixos principais $x$ e $y$, respectivamente, e o termo $N_{e z}$ a força axial de flambagem global elástica por torção pura, como mencionado anteriormente. As variáveis $K_{x} . L_{x}, K_{y} . L_{y}$ e $K_{z} . L_{z}$ denotam a condição de extremidade considerada, sendo no presente estudo adotada viga bi-apoiada e com empenamento livre, o que conduz a $K_{x}=K_{y}=K_{z}=1$. Logo, o sistema de equações passa a ser representado segundo as Equações [17], [18] e [19]:

$$
N_{e y} \cdot A_{1}+M_{e} \cdot \cos \theta \cdot A_{3}=0
$$




$$
\begin{gathered}
N_{e x} \cdot A_{2}-M_{e} \cdot \operatorname{sen} \theta \cdot A_{3}=0 \\
M_{e} \cdot \cos \theta \cdot A_{1}-M_{e} \cdot \operatorname{sen} \theta \cdot A_{2}+r_{0}^{2} \cdot N_{e z} \cdot A_{3}=0
\end{gathered}
$$

Montando-se o determinante do sistema formado pelas equações anteriores, as constantes $A_{1}, A_{2}$ e $A_{3}$ desaparecem, restando como incógnita apenas o valor do momento crítico global $M_{e}$. Igualando a zero e expandindo o determinante, o resultado é a equação do segundo grau apresentada pela Equação [20]:

$$
\left[N_{e x} \cdot \cos ^{2} \theta+N_{e y} \cdot \operatorname{sen}^{2} \theta\right] M_{e}^{2}-r_{0}^{2}\left(N_{e x} \cdot N_{e y} \cdot N_{e z}\right)=0
$$

Apesar do caráter de equação quadrática, para o caso particular de seções duplamente simétricas e ponto-simétricas, as duas soluções da Equação [20] serão iguais em valor absoluto para ambos sentidos de flexão de um mesmo eixo centroidal, independente do eixo de solicitação, isto é, qualquer que seja o ângulo $\theta$ formado entre o vetor momento fletor aplicado e o eixo principal máximo. Com isso, isolando-se a incógnita $M_{e}$, tem-se como solução final para o momento crítico global de seções duplamente simétricas e ponto-simétricas, sob a condição de flexão livre (oblíqua), a Equação [21].

$$
M_{e}=\frac{r_{0}\left(N_{e x} \cdot N_{e y} \cdot N_{e z}\right)^{0,5}}{\left(N_{e x} \cdot \cos ^{2} \theta+N_{e y} \cdot \operatorname{sen}^{2} \theta\right)^{0,5}}
$$

É importante lembrar que esta equação não pode ser aplicada no caso de flexão em seções monossimétricas, visto que as proposições anteriores são válidas somente para o caso particular em que a posição do centro de torção coincide com a posição do centróide da seção, caso este que não ocorre em seções monossimétricas.

A Equação [21], que se mostra simples e prática, foi validada posteriormente com o auxílio do programa CUFSM v.3.12, usado ao longo de todo o estudo, e adicionalmente com a contribuição do programa computacional GBTUL v.2.0, desenvolvido por BEBIANO et al (2010) e representante do método da Teoria Generalizada de Vigas (GBT). Os resultados obtidos pelas soluções analítica e numéricas foram coerentes, apresentando diferença percentual inferior a $10 \%$, diferença essa justificada pelos diferentes métodos de resolução adotados na comparação. 
Considerando ainda a Equação [21], e levando-se em conta o uso de seções pontosimétricas $Z$, simples e enrijecidas, sob flexão em torno do eixo perpendicular à alma, foi elaborada a Tabela 2 reunindo os diferentes valores para o ângulo $\theta$, em graus, para as relações $b_{f} / b_{w}$ e $D / b_{w}$ mais usuais na prática. Para o caso dos perfis enrijecidos os ângulos foram obtidos adotando-se perfis com enrijecedores a $90^{\circ}$, entretanto, conservadoramente, podem ser adotados igualmente para enrijecedores a $45^{\circ}$ (diferenças podem ser consideradas desprezíveis para efeito de cálculo de $M_{e}$ ).

Tabela 2 - Valores de $\theta$, em graus, para seções ponto-simétricas $Z$, simples e enrijecida, sob flexão livre (oblíqua) em torno do eixo perpendicular à alma

\begin{tabular}{|c|c|c|c|c|c|c|c|}
\hline \multirow{2}{*}{$\mathbf{b}_{\mathbf{f}} / \mathbf{b}_{\mathbf{w}}$} & \multicolumn{7}{|c|}{$\mathbf{D} / \mathbf{b}_{\mathbf{w}}$} \\
\cline { 2 - 8 } & $\mathbf{0 , 0 0}$ & $\mathbf{0 , 0 5}$ & $\mathbf{0 , 1 0}$ & $\mathbf{0 , 1 5}$ & $\mathbf{0 , 2 0}$ & $\mathbf{0 , 2 5}$ & $\mathbf{0 , 3 0}$ \\
\hline $\mathbf{0 , 2}$ & 6,33 & 8,35 & 9,92 & 11,20 & 12,29 & 13,23 & 14,06 \\
\hline $\mathbf{0 , 3}$ & 11,34 & 13,68 & 15,58 & 17,20 & 18,62 & 19,89 & 21,03 \\
\hline $\mathbf{0 , 4}$ & 16,81 & 19,34 & 21,47 & 23,32 & 24,98 & 26,50 & 27,91 \\
\hline $\mathbf{0 , 5}$ & 22,50 & 25,12 & 27,35 & 29,33 & 31,14 & 32,83 & 34,42 \\
\hline $\mathbf{0 , 6}$ & 28,19 & 30,78 & 33,02 & 35,03 & 36,88 & 38,63 & 40,30 \\
\hline $\mathbf{0 , 7}$ & 33,66 & 36,12 & 38,27 & 40,22 & 42,04 & 43,76 & 45,41 \\
\hline $\mathbf{0 , 8}$ & 38,74 & 41,00 & 43,00 & 44,84 & 46,55 & 48,19 & 49,77 \\
\hline $\mathbf{0 , 9}$ & 43,32 & 45,35 & 47,17 & 48,86 & 50,44 & 51,97 & 53,44 \\
\hline $\mathbf{1 , 0}$ & 47,38 & 49,17 & 50,81 & 52,33 & 53,78 & 55,18 & 56,54 \\
\hline
\end{tabular}

A relação $\mathrm{D} / \mathrm{b}_{\mathrm{w}}$ igual a zero representa os perfis ponto-simétricos $\mathrm{Z}$ simples (não enrijecidos) Para valores intermediários é sugerido interpolar linearmente

Da mesma maneira que foi adotado para o modo de flambagem local, quando foram disponibilizados os valores dos coeficientes de flambagem $k$, por meio de tabela e expressões matemáticas, para os valores de $\theta$ também foi definida uma expressão, representada pela Equação [22]. Devido à variação quase constante entre os valores do ângulo $\theta$ para cada relação geométrica, foi possível obter uma única expressão para representar os perfis simples e enrijecidos simultaneamente, sem com isso perder em precisão. Novamente a variável $X$ representa a relação $b_{f} / b_{w}$, e $Y$ a relação $D / b_{w}$.

$$
\theta=-9,39+42,24 Y-33,60 Y^{2}+75,94 X+4,13 X Y-19,97 X^{2}
$$

Diante das exposições apresentadas até aqui foram analisadas as Equações [5] e [21], representando, respectivamente, a solução proposta pela ABNT NBR 14762:2010 e a solução desenvolvida no presente estudo para o cálculo de $M_{e}$ em seções ponto- 
simétricas Z. Nota-se que a equação da norma considera o eixo normal à alma dessas seções como principal de inércia máximo, similar à condição de flexão restringida, aplicando-se ainda um fator de redução igual a 0,5 à equação. A razão para a presença desse fator na composição da Equação [5], assim como da formulação da própria equação, foi investigada, contudo não foram encontradas referências literárias que justificassem sua existência, apesar de estar presente nos procedimentos normativos. Já a Equação [21] foi elaborada com base na Teoria da Estabilidade Elástica. A diferença de resultados entre ambas as equações será mensurada na próxima seção.

\section{Comparativo entre os diferentes modos de obtenção de $M_{e}$}

Concluindo a investigação do cálculo do momento fletor crítico de flambagem global elástica de seções ponto-simétricas Z apresentada no presente artigo, foi elaborada a Tabela 3, constando os valores obtidos para $M_{e}$ segundo os diferentes modos discutidos: (i) Equação [5], segundo a ABNT NBR 14762:2010; (ii) Equação [21], solução analítica baseada na Teoria da Estabilidade Elástica; (iii) CUFSM v.3.12 e GBTUL v.2.0, soluções numéricas baseadas no MFF e GBT, respectivamente. A tabela apresenta, ainda, comparativo entre os valores obtidos pela equação da norma brasileira com os valores encontrados pelas demais soluções abordadas, analítica e numéricas. Foram adotados: $b_{w}=100 \mathrm{~mm}, L=4000 \mathrm{~mm}, t=1,0 \mathrm{~mm}, D / b_{w}=0,2, C_{b}=1,0, E=200 \mathrm{GPa}, v=0,3$.

Tabela 3 - Comparativo dos valores obtidos para $M_{e}$, em N.mm, segundo Equação [5] da ABNT NBR 14762:2010, Equação [21] do presente artigo e soluções numéricas

\begin{tabular}{|c|c|c|c|c|c|c|c|}
\hline \multicolumn{2}{|c|}{ Flexão livre (oblíqua) em torno do eixo perpendicular à alma de seções ponto-simétricas $\mathbf{Z}_{\mathbf{9 0}}$} \\
\hline \multirow{2}{*}{$\mathbf{b}_{\mathbf{f}} / \mathbf{b}_{\mathbf{w}}$} & ABNT NBR & \multicolumn{2}{|c|}{ Solução analítica } & \multicolumn{3}{c|}{ Soluções numéricas } \\
\cline { 2 - 8 } & $\mathbf{1 4 7 6 2 : 2 0 1 0}$ & Estabilidade Elástica & \multicolumn{2}{c|}{ MFF } & \multicolumn{3}{c|}{ GBT } \\
\cline { 2 - 8 } & Equação [5] & Equação [21] & dif. (\%) & CUFSM v.3.12 & dif. (\%) & GBTUL v.2.0 & dif. (\%) \\
\hline $\mathbf{0 , 2}$ & 56284 & 115104 & 104,5 & 123978 & 120,3 & 115195 & 104,7 \\
\hline $\mathbf{0 , 3}$ & 113340 & 238275 & 110,2 & 258595 & 128,2 & 238514 & 110,4 \\
\hline $\mathbf{0 , 4}$ & 191760 & 418795 & 118,4 & 456368 & 138,0 & 419082 & 118,5 \\
\hline $\mathbf{0 , 5}$ & 289880 & 663756 & 129,0 & 725005 & 150,1 & 664487 & 129,2 \\
\hline $\mathbf{0 , 6}$ & 405213 & 979789 & 141,8 & 1071753 & 164,5 & 980862 & 142,1 \\
\hline $\mathbf{0 , 7}$ & 535298 & 1373349 & 156,6 & 1503680 & 180,9 & 1374699 & 156,8 \\
\hline $\mathbf{0 , 8}$ & 678161 & 1850809 & 172,9 & 2027766 & 199,0 & 1852522 & 173,2 \\
\hline $\mathbf{0 , 9}$ & 832407 & 2418496 & 190,5 & 2650939 & 218,5 & 2421227 & 190,9 \\
\hline $\mathbf{1 , 0}$ & 997128 & 3082702 & 209,2 & 3380086 & 239,0 & 3086921 & 209,6 \\
\hline
\end{tabular}


De acordo com os resultados apresentados na Tabela 3, percebe-se que o cálculo do momento crítico global $M_{e}$, segundo cada modo investigado, conduz a valores muito distintos, sendo para a solução da ABNT NBR 14762:2010, representada pela Equação [5], sempre inferior aos demais. Logo, em se tratando do dimensionamento estrutural de PFF, onde o valor do momento crítico afeta diretamente o valor do esforço resistente da barra na flexão, a adoção da solução proposta pela norma para o cálculo de $M_{e}$ resultará em um momento fletor resistente muito abaixo daquele esperado, visto que as diferenças obtidas da comparação entre os modos de obtenção de $M_{e}$ foram muito elevadas, principalmente para perfis de comprimento longo, onde o modo de flambagem global é dominante sobre os demais.

É possível observar também como as diferenças percentuais se comportam com a variação da relação $b_{f} / b_{w}$. Nota-se um aumento das diferenças à medida que a relação $b_{f} / b_{w}$ também aumenta. A observação é válida quando se comparam os resultados obtidos pela Equação [5] da ABNT NBR 14762:2010 com os resultados da Equação [21] e dos programas computacionais CUFSM v.3.12 e GBTUL v.2.0.

Analisando os valores obtidos da Equação [21] frente aos do programa GBTUL v.2.0, notam-se diferenças irrelevantes, muito abaixo de 1\%, o que corrobora a eficiência da solução analítica apresentada no presente estudo. Comparando-se aos do programa CUFSM v.3.12 as diferenças são um pouco mais expressivas, mas ainda assim ficam abaixo de $10 \%$, justificadas conforme o motivo mencionado na seção anterior.

Apesar dos resultados da Tabela 3 estarem associados a parâmetros específicos como espessura $t$ igual a $1 \mathrm{~mm}$, comprimento $L$ igual a $4000 \mathrm{~mm}$ e relação $D / b_{w}$ igual a 0,2 , verificou-se que a variação dessas propriedades pouco altera as diferenças percentuais obtidas da comparação entre os resultados das equações propostas, isto é, quando considerados perfis com diferentes espessura, comprimento e largura do enrijecedor de borda. Logo, conclui-se que as seções analisadas bastam para justificar a solução proposta pela Equação [21]. A afirmação baseia-se em testes efetuados pelos autores, porém não registrados neste artigo, a fim de não estender o conteúdo do mesmo. 


\section{Conclusões}

A presente pesquisa permitiu concluir que as prescrições da norma brasileira ABNT NBR 14762:2010 deixam lacunas quanto ao dimensionamento de seções ponto-simétricas Z sob flexão simples em torno do eixo perpendicular à alma, em se tratando dos modos de flambagem local e global.

Para o modo de flambagem local, notou-se a ausência de prescrições para cálculo do coeficiente de flambagem local $k$ para seção completa sob a condição de flexão oblíqua. Há apenas indicações para o cálculo desse coeficiente para a condição de flexão restringida, a qual foi verificada e confirmada em muito boa concordância frente aos resultados numéricos. No entanto, a norma não esclarece ser essa a condição de flexão a que se refere. Nesse contexto, sugere-se a inclusão das Equações [2] e [3] e Tabela 1 propostas para o cálculo do coeficiente de flambagem local $k$ / para as seções pontosimétricas Z na flexão oblíqua em torno do eixo perpendicular à alma da seção.

O estudo do modo de flambagem global apresentou resultados em desacordo com a Teoria da Estabilidade Elástica, visto que a equação de cálculo do momento crítico global constante na norma brasileira não está em concordância com a teoria apresentada por TIMOSHENKO e GERE (1961). A solução proposta pela ABNT NBR 14762:2010 demonstrou ser muito antieconômica, com diferenças podendo chegar a $200 \%$ inferiores ao valor esperado. Considerando que a Equação [21] elaborada na pesquisa foi validada pela comparação com resultados obtidos pela solução numérica da flambagem segundo os programas CUFSM v.3.12 e GBTUL v.2.0, seria recomendável uma validação experimental posterior, visando assim uma possível substituição da Equação [5] constante na ABNT NBR 14762:2010 pela Equação [21] desenvolvida na presente pesquisa em uma próxima revisão da norma.

\section{Agradecimentos}

O primeiro autor agradece o apoio financeiro da Coordenação de Aperfeiçoamento de Pessoal de Nível Superior (CAPES) para a realização da presente pesquisa. 


\section{$9 \quad$ Referências Bibliográficas}

ABNT, NBR 14762:2010. Dimensionamento de estruturas de aço constituídas por perfis formados a frio, Associação Brasileira de Normas Técnicas, Rio de Janeiro, RJ. 2010.

AISI, S100-16. North American Specification for the Design of Cold-Formed Steel Structural Members, American Iron and Steel Institute, Washington, DC. 2016.

BATISTA, Eduardo de Miranda. "Effective section method: A general direct method for the design of steel cold-formed members under local-global buckling interaction", Thin-Walled Structures, v.48, pp. 345-356.2010.

BEBIANO, Rui; SILVESTRE, Nuno; CAMOTIM, Dinar. "GBTul $1.0 \beta$ - Buckling and Vibration Analysis of Thin-Walled Members", GBT Theoretical Background, DECivil/IST, Technical University of Lisbon, Portugal. 2010.

FÁVERO NETO, Alomir Hélio. Terças em perfis de aço formados a frio com continuidade nos apoios: ênfase ao estudo das ligações de alma parafusadas com transpasse ou luva, Dissertação de Mestrado, EESC/USP, São Carlos, SP. 2013.

PEREIRA, Vitor Faustino. Integração entre sistemas de cobertura metálica e estrutura de concreto em galpões. Aplicações em sistemas pré-moldados e tilt-up, Apostila, Prof. adjunto UEL. 2016.

SCHAFER, Benjamin William. "Designing Cold-Formed Steel Using the Direct Strength Method", $1^{\text {th }}$ International Specialty Conference on Cold-Formed Steel Structures, Orlando, FL. 2006.

SCHAFER, Benjamin William; ÁDÁNY, Sandor. "Buckling analysis of cold-formed steel members using CUFSM: conventional and constrained finite strip methods", $18^{\text {th }}$ International Specialty Conference on Cold-Formed Steel Structures, Orlando, FL. 2006.

TIMOSHENKO, Stephen Prokofievich; GERE, James Monroe. Theory of Elastic Stability, 2 ed., McGraw-Hill, New York. 1961. 